\title{
Study on Data Generating Method for Communication Area of Shortwave Communication Radios under Terrain shading
}

\author{
Zhao Yuan, Zhang Kun \\ National University of Defense Technology electronic warfare academy, Hefei, china \\ 842068234@qq.com
}

Keywords: Terrain shading; Shortwave communication; Communication area; Data generating

\begin{abstract}
Whether the communication is smooth or not is an important factor that affects intelligence transmission, command, guidance and combat synergy. Calculating the communication area of communication equipment and achieve visualization can well demonstrate the communication efficiency, and guide commanders scientific decision-making. However, the existing communication area calculation model is mainly based on the smooth ground assumption, less consideration of the problem of unblocked communication caused by the terrain shading, and cannot be completely applied the actual combat environment. So, taking shortwave radio as an example, this paper puts forward the method of data generation and visualization in the communication area under the condition of terrain shading, and achieves better results.
\end{abstract}

\section{Introduction}

Shortwave communication usually refers to radio communication using electromagnetic waves having a wavelength of $10-100 \mathrm{~m}^{[1]}$. Shortwave radios have played an important role in military communication due to their simple equipment, low cost, convenient assumptions and flexible maneuvers since they came out ${ }^{[2]}$. The basic modes of shortwave propagation include ground wave propagation and sky wave propagation. Among them, the ground wave mode of propagation is close to the ground and propagates along the interface between earth and air. The propagation depends on the unevenness of the ground ${ }^{[3]}$ and the electrical properties of the geology ${ }^{[4]}$. The article mainly focuses on the former, analyzes the shortwave ground wave propagation model under the condition of masking, provides a method for calculation and simulation of the communication area of shortwave radios, and makes it easy for commanders to make accurate judgments and make scientific decisions.

\section{Visibility Analysis Theory}

The principle of visibility analysis is based on the judgment between point to point. In the use of computer to determine the visibility, using the calculation method, the principle is as follows:

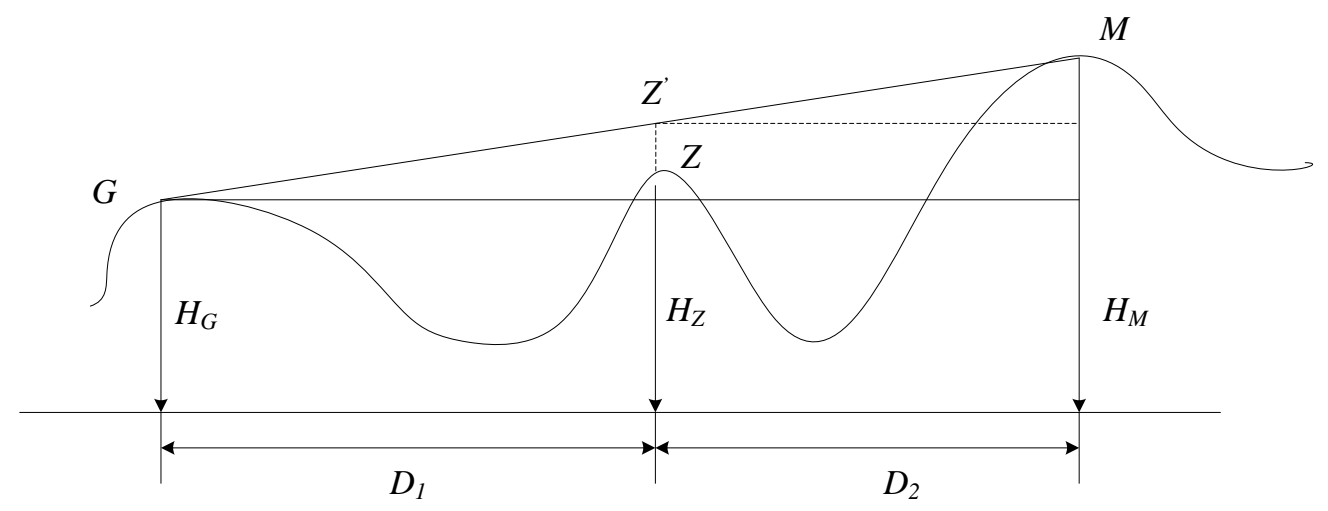

Figure.1 terrain shading diagram 
As shown in figure.1: let $\mathrm{G}, \mathrm{M}, \mathrm{Z}$ be the observation point, the target point and the shadowing point respectively, and their elevation are $\mathrm{H}_{G}, \mathrm{H}_{M}$ and $\mathrm{H}_{\mathrm{Z}}$ respectively. The distance between the observation point and the shadowing point is $\mathrm{D}_{1}$, and the distance between the target point and the shadowing point is $\mathrm{D}_{2}$.

Set the shadowing point $Z$ along the vertical extension of the intersection of the horizon and the point $Z^{\prime}$, and this is called the hypothetical shading point. If we ascertain the elevation $H^{\prime} Z$, when $H^{\prime} Z>H Z$,it must be visible.

\section{Shortwave Communication Distance Calculation Model}

\subsection{Shortwave Communication Distance Calculation Model when there's no shades}

Literature [5][6] proposed that when the radio wave is transmitted planely, the maximum communication distance satisfies:

$$
P_{t} G_{t}\left(\frac{\lambda}{4 \pi R_{\max }}\right)^{2} q_{r t} W^{2}=P_{r(\min )}
$$

In where, $P_{t}$ is the transmitting signal power of the shortwave communication station; $G_{t}$ is the gain of the signal transmitting antenna in the receiving equipment direction; $q_{r t}$ is the gain of the receiving antenna in the direction of the signal transmitting station; $\lambda$ is the wavelength of the communication signal; $P_{r(\min )}$ is the minimum detectable signal power of the communication receiver; $W$ is the Ground attenuation factor.

The ground attenuation factor can be expressed as follows ${ }^{[7]}$ :

$$
W=\frac{2+0.3 x}{2+x+0.6 x^{2}}
$$

In where, $x$ is Middle amount

$$
x=\frac{\pi R_{\max }}{\lambda} \frac{\sqrt{(\varepsilon-1)+(60 \lambda \sigma)^{2}}}{\varepsilon^{\prime 2}+(60 \lambda \sigma)^{2}}
$$

In where,$\varepsilon$ is the relative permittivity of the ground; $\sigma$ is the ground conductivity.

\subsection{Calculation Model of Communication Distance under Terrain Shading}

The main assumption is that the terrain obscuring model assumptions when the mountain block (Figure 1), assuming that the height of the mountain $h$.

\subsubsection{Only a single shade.}

If the shadow height is greater than 2 times the wavelength, that is $h \geqslant 2 \lambda$, it is blocked. If the shadow height is less than 2 times the wavelength, that is $h<2 \lambda$,it can diffraction, and diffraction loss is $10 \lg (n+1) 2$, that is, the equivalent transmit power of $1 / 4$ of the original power after blocking.

\subsubsection{If there are multiple shades, it is simplified as a sequentially diffracted single masking}

If there are $m$ shielding blocks in the azimuth, the next peak $(1 \leqslant i \leqslant m)$ is removed in turn, and the calculation model of the communication distance $R$ is as follows:

1) Calculation $h_{i} / \lambda$;

2) Calculate the distance between the i-1and i mountain;

3) When the i mountain blocks, basing on Power equivalence principle of diffraction loss,we can calculate the propagation distance $R_{i}$ after the wave passes over the i mountain. 


\subsubsection{The calculation model is as follows:}

\begin{tabular}{|c|c|c|}
\hline $\begin{array}{l}\text { A sketch map of the shading of the } \\
\text { mountain }\end{array}$ & $\begin{array}{l}\text { Description of the } \\
\text { situation }\end{array}$ & $\begin{array}{c}\text { Communicati } \\
\text { on distance } \\
\text { calculation }\end{array}$ \\
\hline$\frac{r_{i}}{r_{R_{i-1}}}$ & $\begin{array}{l}\text { When } h_{i} / \lambda \geqslant 2 \text {, } \\
r_{i}>R_{i-1} \text {,it can't } \\
\text { spread to the } \mathrm{i} \\
\text { mountain ; End of } \\
\text { calculation }\end{array}$ & $\begin{array}{l}\text { Assuming } \\
\text { thecommunic } \\
\text { ationdistance } \\
\text { after the } \\
\text { wave passes } \\
\text { over the } \\
i-1 \\
\text { mountain, } \\
\text { then } \\
R=R_{n}+R_{i-1}\end{array}$ \\
\hline$\frac{r_{i}}{R_{t-1}}$ & $\begin{array}{l}\text { When } h_{i} / \lambda \geqslant 2 \text {, } \\
\qquad r_{i} \leqslant R_{i-1} \text {,it is } \\
\text { blocked ; End of } \\
\quad \text { calculation }\end{array}$ & $R=R_{n}+r_{i}$ \\
\hline$\frac{r_{i}}{r_{R_{i-1}}}$ & $\begin{array}{l}\text { When } h_{i} / \lambda<2 \text {, } \\
r_{i} \leqslant R_{i-1} \text { it can't } \\
\text { spread to the } \mathrm{i} \\
\text { mountain ; End of } \\
\text { calculation }\end{array}$ & $R=R_{n}+R_{i-1}$ \\
\hline$\frac{r_{i}}{R_{i-1}}$ & $\begin{array}{l}\text { When } h_{i} / \lambda<2 \text {, } \\
r_{i} \leqslant R_{i-1}, \text { Then the } \\
\text { next mountain } \\
\text { shading continues } \\
\text { to be calculated } \\
\text { until the above } \\
\text { three cases appear. }\end{array}$ & $R=R_{n}+r_{i}+\ldots$ \\
\hline
\end{tabular}

Figure.2 Propagation and calculation diagram

\section{Simulation}

\subsection{Computer simulation steps}

The principle of using computer simulation is as follows: a single HF communication station is used as the observation point, the maximum communication distance is the observation radius, the omnidirectional angle is used to calculate the communication distance under the terrain shadowing conditions, and the data is collected and drawn according to the azimuth and the corresponding 
communication distance Out of shortwave communications terrain under the conditions of the terrain masking communication smooth area image. Specific steps are as follows:

Step1: Load a high-resolution map to get the deployment location of the communication station;

Step2: Using the unobstructed short-wave communication distance calculation model, calculate the maximum communication distance without shielding.

Step3: Taking the position of the communication station as the center, the maximum communication distance is a radius, the horizontal azimuth angle is sequentially masked and analyzed from $0-360^{\circ}$, and the communication distance of each azimuth is calculated $R$ by using the communication distance calculation model under the shielding condition.

Step4: Triangular fan for drawing basic elements, according to the data obtained to draw the smooth area graphics.

\subsection{Data sampling}

Based on a high-resolution map, a short-wave communications station was deployed somewhere using the proposed solution and the maximum communication distance calculated using the function was 124884 meters. Follow the above steps to get the full range of communication distance. Figure.3 lists the data $0-100^{\circ}$, Figure 4 According to the read $360^{\circ}$ azimuth data, using excel to draw the communication unobstructed area graphics to verify the correctness of access to data.

\begin{tabular}{|r|r|r|r|r|r|r|r|}
\hline 0 & 37884.48368 & 40 & 6818.90773 & 60 & 124884 & 80 & 124884 \\
\hline 1 & 30811.51252 & 41 & 7816.24243 & 61 & 124884 & 81 & 124884 \\
\hline 2 & 46241.62691 & 42 & 124884 & 62 & 124884 & 82 & 124884 \\
\hline 3 & 30664.30707 & 43 & 124884 & 63 & 124884 & 83 & 124884 \\
\hline 4 & 7703.123199 & 44 & 28871.64221 & 64 & 124884 & 84 & 124884 \\
\hline 5 & 7803.045185 & 45 & 28341.87707 & 65 & 124884 & 85 & 124884 \\
\hline 6 & 15747.91499 & 46 & 28187.2836 & 66 & 124884 & 86 & 124884 \\
\hline 7 & 8048.136834 & 47 & 124884 & 67 & 124884 & 87 & 124884 \\
\hline 8 & 32928.82568 & 48 & 124884 & 68 & 124884 & 88 & 124884 \\
\hline 9 & 8497.191808 & 49 & 124884 & 69 & 124884 & 89 & 124884 \\
\hline 10 & 41506.31804 & 50 & 124884 & 70 & 124884 & 90 & 124884 \\
\hline 11 & 6641.687539 & 51 & 124884 & 71 & 124884 & 91 & 124884 \\
\hline 12 & 6494.632479 & 52 & 124884 & 72 & 124884 & 92 & 124884 \\
\hline 13 & 6225.031519 & 53 & 45497.7319 & 73 & 124884 & 93 & 124884 \\
\hline 14 & 5998.792935 & 54 & 34687.71924 & 74 & 124884 & 94 & 124884 \\
\hline 15 & 5938.462643 & 55 & 43163.81478 & 75 & 124884 & 95 & 124884 \\
\hline 16 & 5881.902994 & 56 & 124884 & 76 & 124884 & 96 & 124884 \\
\hline 17 & 5827.228666 & 57 & 124884 & 77 & 124884 & 97 & 124884 \\
\hline 18 & 5776.324979 & 58 & 124884 & 78 & 124884 & 98 & 124884 \\
\hline 19 & 5727.306614 & 59 & 124884 & 79 & 124884 & 99 & 124884 \\
\hline
\end{tabular}

Figure.3 Data sampling diagram

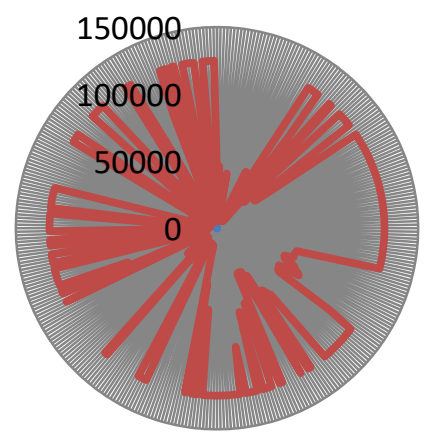

Figure.4 excel drawings 


\subsection{Draw method}

Drawing shortwave communication station communication unobstructed graphics, the sampling of the azimuth and the corresponding communication distance is transmitted to the rendering end of the simulation system, the triangle fan for drawing basic elements, the adjacent azimuth will be the border point and the radio connection, get A triangular fan (Figure.5. b). And so on, traversing the azimuth, you can get the entire area, to achieve the visual communication of unobstructed area. Simulation results shown in Figure.5. b. The figure is basically the same as Figure 6.a, indicating that the data transmission and drawing process are both feasible.

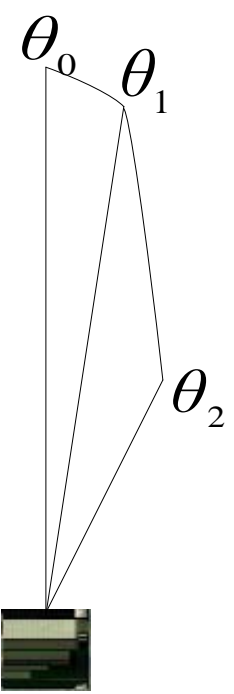

Figure.5.a Triangle fan rendering diagram

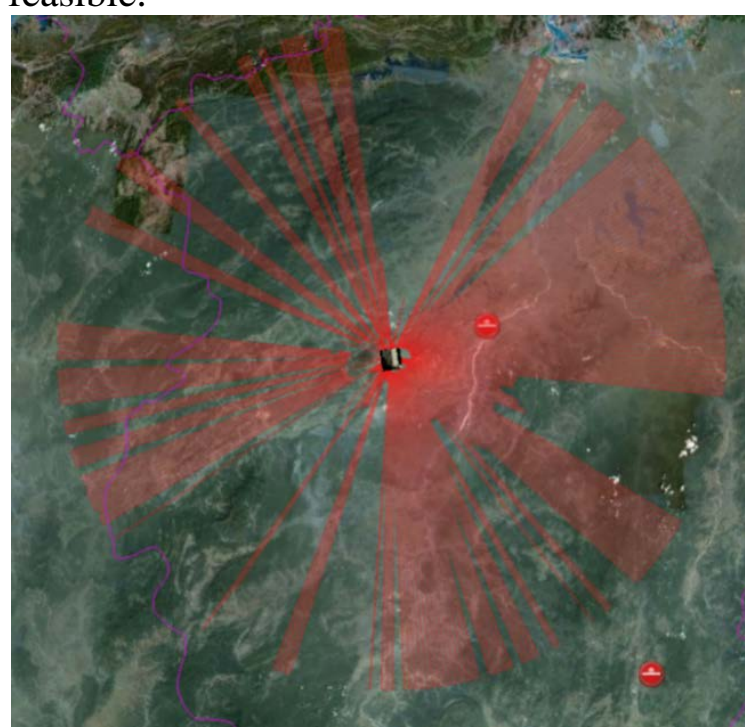

Figure.5.b Achieve renderings

Figure 5

\section{Conclusion}

Obtained by computer simulation of short-wave communication under cover conditions unobstructed area, is based on the location of the communication station as the center of the communication distance $R_{\max }$ (no shaded) or the $R$ (shaded) vertex envelope surrounded by a collection of internal regions. Through the simulation and analysis of the unobstructed communication area of a single shortwave radio station, it is found that the visualization shows the communication effect of stations in different deployment locations well and can effectively guide the commanders in the deployment of the radio and the receiver, facilitating the quick judgment of the commander, decision making.

\section{References}

[1] Ning Zhang, “Talking about the Influencing Factors of Shortwave Ground Wave Propagation”, Digital communication world, 2013

[2] Tang-Wei Liu, "Analysis of the role and characteristics of shortwave in radio communications”, Digital communication world, 2017

[3] Zheng Song, “The Antenna and the Radio Waves Propagation”, Xidian University press, 2011

[4] Hai-peng Fu, “Analysis of Ground Wave Propagation under Different Geological Conditions”, Communication confrontation, 2007, pp. 47-50

[5] Guo-pei Shao, "Electronic Countermeasure Combat Effectiveness Analysis”, The people's liberation army press, 1998

[6] Guo-pei Shao, “Electronic Countermeasure Tactical Calculation Method”, The people's liberation army press, 2010

[7] Jin-bai Mo, "Shortwave ground wave propagation path loss analysis”, Communication world, 2016 